\title{
An Improved Image Restoration and Edge Detection Technique
}

\author{
Orvila Sarker", Rokeya Begum Jothi
}

Department of Information and Communication Technology, Comilla University, BANGLADESH

Corresponding Contact:

Email: orvila.sarker@gmail.com

\begin{abstract}
Clustering and edge based segmentation are two basic image segmentation technique. This paper involves image clustering based restoration technique for finding out the set of consequential groups and restoring the original image from a noisy image. Previously, the feature of image cluster computing and restoration method is researched separately but now we combined the cluster and restoration method together. The $\mathrm{k}$ means clustering algorithm is applied on similar objects to create a cluster that separate noisy pixels and finally we use Gaussian filter to restore the noise corrupted image which enhanced the image quality. The simulation results show that the techniques are able to produce better output in terms of contrast and resolution. In case of edge based segmentation, canny edge detection algorithm is the optimal one because of its low error rate, good localization, only one response to a single edge etc. In this work, we have showed that applying double threshold in canny edge detection algorithm provides reasonably better output.
\end{abstract}

Key words

Clustering, K-means algorithm, Restoration, Segmentation, Edge detection, Canny edge detection algorithm, Threshold

This article is is licensed under a Creative Commons Attribution-NonCommercial 4.0 International License. Attribution-NonCommercial (CC BY-NC) license lets others remix, tweak, and build upon work non-commercially, and although the new works must also acknowledge \& be non-commercial.

\section{INTRODUCTION}

A digital image is defined as a composition of finite number of square elements referred to as pixels. Each of these pixels has a specific location and value (Mishra \& Sharma, 2014).Three basic types of digital image are Binary image, Gray scale image and Color image. Binary image consist of only two values, 0 and 1, for each pixel. Gray-scale images have values ranging from 0 to 255, where 0 indicates black color, 255 indicates white color and the other values in between 0 and 255 represents shades of gray. An array in two dimensions of RGB (Green, Red and Blue) triples is known as color image. Basically in a triple, each number lies in between 0 to 255, where 0 shows the absence of any primary 
color in that pixel and 255 shows a maximum amount of that prime color in that pixel (Sharma \& Suji, 2016).

In the field of image processing, image segmentation and image restoration plays a significant role for meaningful analysis of an image. Image segmentation is the process in which a digital image is broken down into small parts (Nirgude \& Jain, 2014). Different image segmentation techniques are Region based, Edge based, and Threshold based and Clustering based Segmentation. In Region based segmentation technique, an image is split into many unlike sections based on object, color or intensity etc. Edge detection refers to the process of identifying and locating sharp discontinuities in an image (Maini \& Aggarwal). The edge based image segmentation is done by noticing pixels or edges in between diverse sections that basically have quick change in intensity and the outcome is an image in binary form. The edges have been used to measure the size of objects in an image; to isolate particular objects from their background; to recognize or classify objects (Kumar \& Arthanariee, 2013). Threshold based image segmentation is a method used for discriminating background from foreground (Sharma \& Suji, 2016). In threshold based segmentation, the image histogram can be partitioned into two classes using a single value (called bi-level thresholding) or multiple classes using multiple values(called multi-level thresholding) based on the characteristics of the histogram. In the bi-level thresholding, pixels with intensity values less than the threshold are set as background while others are set as object. In multiple-level thresholding, pixels with the intensity values between two successive thresholds are assigned as a class (Fatma \& Sharma, 2014). Clustering based segmentation refers to the process of grouping samples so that the samples are similar within each group (Tayal \& Raghuwanshi, 2011). An image can be segmented based on its keyword (metadata) or its content (description) (Sharma \& Suji, 2016).

Image restoration is the process of retrieving the original image from the noisy image that is degraded or blurred by some external sources. Noise may be added to an image by the effect of different lighting condition, atmospheric turbulence, poor focus and motion between camera and original image etc. Generally when intensity of an image point is spread over several pixels, it goes blurred and the noise doesn't remain uniform over an entire image. The maximum amount of blur in the image is at the center of the image. So an efficient way of restoration would be to denoise the center part of an image compared to corner segments. In the field of image processing, several techniques for image restoration has been proposed. Each technique restores the image having different type of blur. Some techniques restore the Gaussian blur, some restore the motion blur and some restore the out of focus blur. Clustering process provides a way to cluster a dataset into different sub-clusters based on their common property. Clustering algorithm follows the nearest neighbor search algorithm for creating the clusters or classes. The elements in the same class exhibit the same property. In each class, different similarity measures are used to decide which member belongs to the corresponding class (Saxena \& Kumar, 2014).

In this paper, we investigated image restoration process, detect unwanted objects by using the cluster computation.We apply k-means clustering algorithm to group unwanted noise pixels based on color, texture and gray levels.The task of debluring, a form of image restoration is performed to obtain the original, sharp version of a blurred image.

Also, we have analyzed canny edge detection algorithm to detect edges of an input image by applying double threshold. It has been showed that the application of double threshold provides better edge detection and a more clear form of input image. 


\section{MetHodology}

\section{Basic $k$ - means clustering algorithm}

It is an iterative technique that is used to partition an image into k clusters. It assigns each point to the cluster whose centre is most nearest. The centre is the average of all the points in the cluster. For example if the data set has three dimensions and the cluster has two points $\mathrm{X}=\left(\mathrm{x}_{1}, \mathrm{x}_{2}, \mathrm{x}_{3}\right)$ and $\mathrm{Y}=\left(\mathrm{y}_{1}, \mathrm{y}_{2}, \mathrm{y}_{3}\right)$, then centroid becomes $\mathrm{Z}=\left(\mathrm{z}_{1}, \mathrm{Z}_{2}, \mathrm{Z}_{3}\right)$ where $\mathrm{z}_{1}=$ $\left(\mathrm{x}_{1}+\mathrm{y}_{1}\right) / 2, \mathrm{z}_{2}=\left(\mathrm{x}_{2}+\mathrm{y}_{2}\right) / 2$ and $\mathrm{z}_{3}=\left(\mathrm{x}_{3}+\mathrm{y}_{3}\right) / 2$. Figure 1 shows $\mathrm{K}$-means clustering for $\mathrm{k}=4$. The algorithm steps are:

- Choose the number of clusters, $\mathrm{k}$.

- Randomly generate $\mathrm{k}$ clusters and determine the cluster centroid.

- Assign each point to the nearest cluster centroid.

- Recompute the new cluster centroid.

- Repeat steps 3 and 4 until some convergence criterion is met.

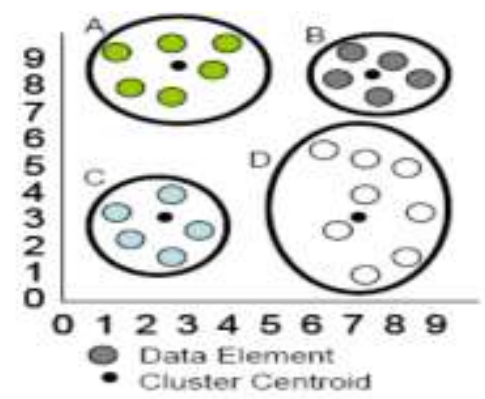

Figure 1. K-means clustering in two dimensions for $\mathrm{k}=4$ (Patel et. al., 2013)

Steps involved in proposed restoration technique using $k$-means clustering

We use k-means clustering algorithm to group original and noise pixels and MATLAB simulator to perform the algorithm. The algorithm steps are:

- Read an image into MATLAB environment.

- Display the image read.

- Consider a pixel say im(i,j) and identify its nearest neighbours. Take all the eight neighbours for chessboard distance and four for block distance.

- Extract a sub matrix containing the elements of im(i,j).

- Calculate the mean value of all the neighbours of the sub matrix.

- Approximate the mean value obtained in step 5.

- $\quad$ Replace the pixel at im(i,j) with the value obtained in step 6. Go to step 3.

- Display the restored image.

\section{Improved canny edge detection algorithm}

The canny edge detection algorithm is adaptable to various environment. The proposed process of canny edge detection algorithm can be broken down to 5 different steps with a slight modification:

- Apply Gaussian filter to smooth the image in order to reduce the noise

- Discover the intensity gradients of that image

- Apply non maximum suppression to get rid of simulated response to edge detection 
- Increase the threshold amount to detect the potential edges

- Find edge by hysteresis: finalize the detection of edges by restraining all the other edges that are disabled and not connected to strong edges.

\section{RESULTS AND DISCUSSION}

\section{Analysis of proposed restoration process}

In this paper, a blurred colored image is used for this simulation analysis that is shown in Fig. 2(a). We propose a colored image restoration method which is based on the $\mathrm{k}$ means clustering method to group into a cluster for detecting the noise pixels.

The output clustered image is shown in Fig. 2(b). In this figure, the $X$ axis represents the pixels point that's range is $0-5$ and the $Y$ axis represents the contrast point that's range is 0 5. There is also define the two centroid point that are the two centre point of the clusters. The red mark points are indicated the noisy pixels. The blue mark points are indicated the exact colored pixels of the image. The cluster points are constructed depend on the pixels and contrast of the image. This graphical figure shows that the result of using at most 20 nearest neighbour pixels.

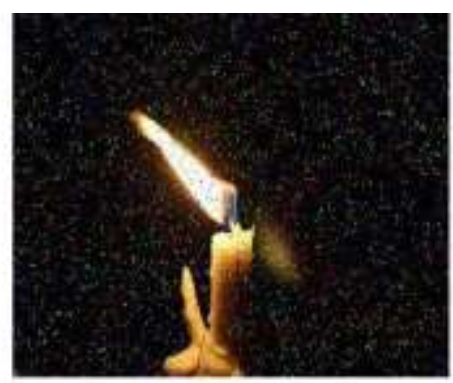

(a)

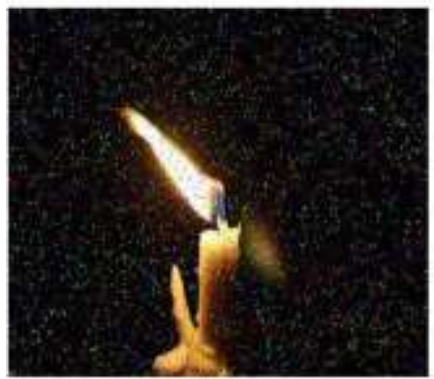

(c)

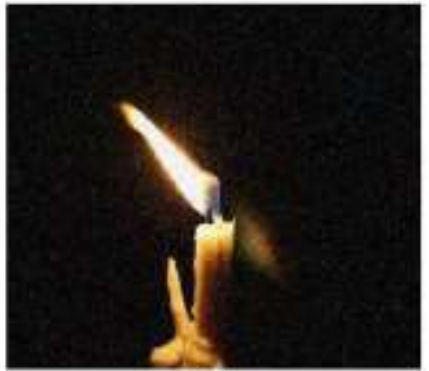

(d)

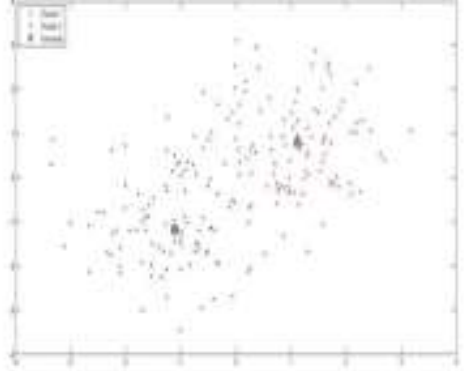

(b)

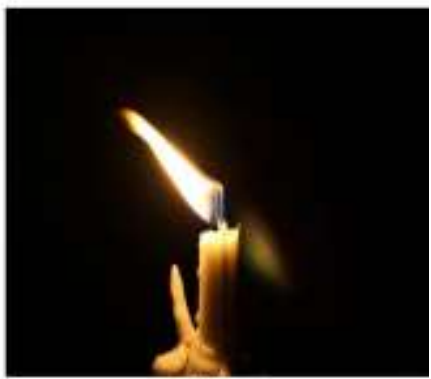

(e)

Figure 2: Proposed restoration process (a) Input Image (b) Clustered Image (c) Reconstructed clustered noisy image (d) Gaussian noise added image (e) Restored image

In Fig. 2(c), we reconstructed the input noisy image. Here, the salt and pepper noise is used. The reconstructed clustered noisy image is shown in Fig. 2(c). We also applied Gaussian noise as shown in Fig. 2(d) and obtained the clearer appearance in Fig. 2(e).

Then we are using the restoration algorithm on that image which is blurred by Gaussian noise and Salt and pepper noise filter, to obtain a restored and clear image that was our original image. The result is shown in Fig. 2(e). The properties of the image are also measured and compared to the before and after restored image and tabulated in table 1. 
Table 1 . Image quality before and after restoration

\begin{tabular}{|c|c|c|}
\hline Parameters & Before Restoration & After Restoration \\
\hline Image contrast & 0.263 & 0.481 \\
\hline Resolution(pixels) & $788^{* 526}$ & $1024^{*} 683$ \\
\hline
\end{tabular}

From Table 1, we observe that the contrast and the resolution of this image is comparatively higher than before the restored image. The contrast is increase in 0.218 and also growth the resolution (pixels) in 284904. Now the appearance of output image is much clear than the before restored image that was mixed up with noised.

\section{Improved edge detection process}

We increased the threshold value in canny edge detection process to improve the edge quality of input image. Fig. 3 illustrate the entire edge detection process. Fig. 3(a) and Fig. 3(b) represent the input images. Fig 3(c) and 3(d) indicates the response after applying canny edge detection algorithm. Finally, Fig. 3(e) and 3(f) shows the improvement after increasing the normal threshold value in canny edge detection algorithm compared to Fig. $3(\mathrm{c})$ and $3(\mathrm{~d})$ respectively. We also show that the appearance of the proposed output image is quite better.

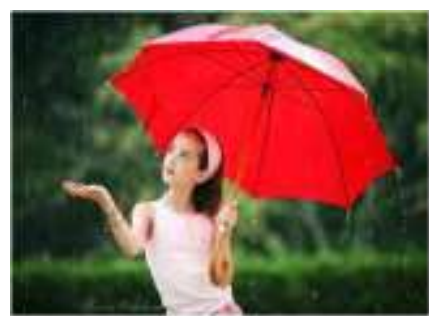

(a)

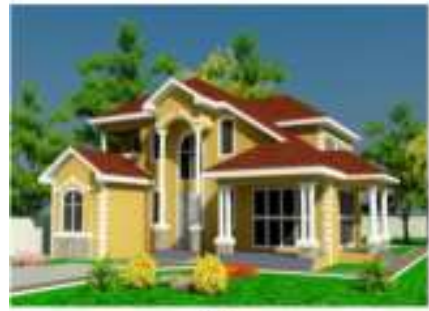

(b)

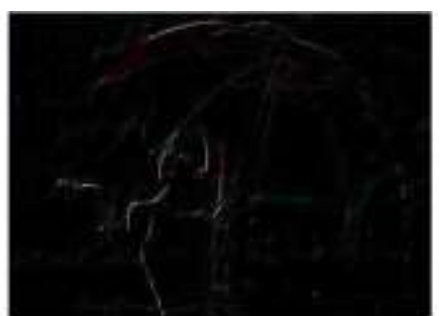

(c)

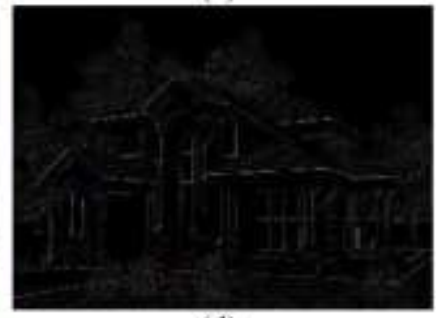

(d)

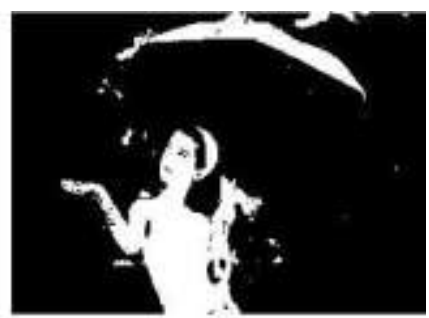

(e)

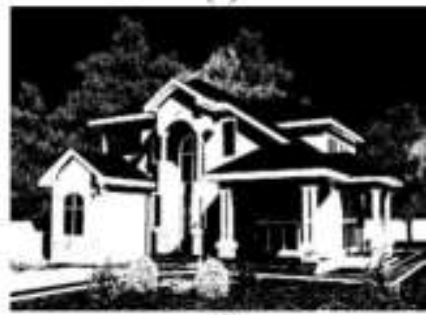

(f)

Figure 3: Performance comparison between canny edge detection and proposed edge detection process (a) Input sample image 1 (b) Input sample image 2 (c) Canny edge detection output for image 1 (d) Canny edge detection output for image 2 (e) Proposed output for image 1 (f) Proposed output for image 2

\section{CONCLUSION}

We have used a k-means clustering algorithm to detect the noise objects then applied the restoration technique to remove the noisy pixels. In terms of contrast and resolution, the restored image gives a better quality. The results depict that the proposed work is good for the existing framework. For such a noisy image, if the background color is unknown it is difficult to restore the original image. In future work, we plan to introduce a new combined method for restoring image. In case of edge detection, changing the threshold value improves the edge quality of the input image which is clearly visible from the simulation results. 


\section{REFERENCES}

Fatma, M. and Sharma, J. (2014)."Leukemia Image Segmentation using K-Means Clustering and HSI Color Image Segmentation". International Journal of Computer Applications. vol. 94, no. 12, pp. 6-9.

Kumar, R. and Arthanariee, A., M. (2013). "A Comparative Study of Image Segmentation Using Edge-Based Approach". International Journal of Mathematical, Computational, Physical, Electrical and Computer Engineering vol. 7, no. 3, pp. 510-514.

Maini, R. and Aggarwal, Dr. H. "Study and Comparison of Various Image Edge Detection Techniques". International Journal of Image Processing (IJIP), International Journal of Image Processing (IJIP), vol. 3, Issue 1, pp.1-12.

Mishra, S., Sharma, M. T. (2014). "Image Restoration Technique for Fog Degraded Image". International Journal of Computer Trends and Technology (IJCTT), vol. 18 no. 5, pp. 208-213.

Nirgude, R., Jain, S. (2014). “Color Image Segmentation with K means clustering and dynamic region margin". Sai Om Journal of Science, Engineering \& Technology. vol. 1, Issue 5, pp. 1-10.

Patel, P. M. and Shah, B. N. and V. Shah. (2013). "Image segmentation using K-mean clustering for finding tumor in medical application". International Journal of Computer Trends and Technology (IJCTT). vol. 4, Issue 5, pp. 1239-1242.

Saxena, S. and Kumar, R. S. (2014). "A Novel Approach of Image Restoration Based on Segmentation and Fuzzy Clustering". International Journal of Signal Processing, Image Processing and Pattern Recognition. vol.7, no. 4, pp.255-264.

Sharma, P., Suji, J. (2016). "A Review on Image Segmentation with its Clustering Techniques". International Journal of Signal Processing, Image Processing and Pattern Recognition vol.9, no.5, pp.209-218.

Tayal, M. A. and Raghuwanshi. (2011). M. M. “Review on Various Clustering Methods for the Image Data". Special Issue Journal of Emerging Trends in Computing and Information sciences, vol. 2, pp. 3438 .

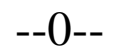

ISSN: 2409-3629

Online Archive Link: $\underline{\text { https://abc.us.org/ojs/index.php/ei/issue/archive }}$ 